\title{
Serum Immunoglobulin G4 is Closely Related to Develop Graves' Ophthalmopathy.
}

\author{
Sung Hoon Yu*, Jae Myung Yu, Hyung Joon Yoo, Seong Jin Lee
}

Division of Endocrinology and Metabolism, Department of Internal Medicine, Hallym University College of Medicine, Seoul, Korea

\section{Introduction}

Grave's ophthalmopathy (GO) is most common extrathyroidal manifestation of Grave's disease (GD). It affects about 25-50 \% of Graves' patients with variable severity (mild, moderate to severe, and sight-threatening). The possible pathogenesis of $\mathrm{GO}$ can be swelling of extraocular muscle and adipose tissue following cytokine induced accumulation of glycosaminoglycans. But we do not know precise mechanism of onset of GO.

In 2001, Hamano et al. first reported lgG4-related disease (IgG4-RD) with regard to autoimmune pancreatitis. IgG4-RD is characterized by hypergammaglubulinemia with elevated IgG4 levels. So, variety of IgG4-RD were proposed, such as Riedel's thyroiditis, Mikulicz's syndrome, retroperitoneal fibrosis, multifocal fibrosclerosis (affecting the orbits, thyroid gland, retroperitoenum and other tissues), periaortitis, peirarteritis, and mediastinal fibrosis. However, there are limited data about the relationship between IgG4 and Graves' ophthalmopathy (GO). In this study, we investivated the association between $\lg G 4$ and $\mathrm{GO}$.

\section{Methods}

\section{Subjects}

This cross-sectional study was performed on subjects who were visited or referred to Endocrinology and Metabolism Outpatient Clinic of the Hallym University Sacred Heart Hospital, Republic of Korea, from January 2013 to August 2015. The patients initially diagnosed as Graves' disease (GD) were enrolled, and thus none of the patients underwent antithyroid drug medication or surgery or radioiodine treatment in the time of enrollment. The diagnosis of GD was based on the presence of hyperthyroidism, positive TSH receptor antibody (TR Ab), and/or increased ${ }^{131}$ I thyroid uptake. GD patients were divided into two groups depending on the presence of Graves' ophthalmopathy (GO) on the basis of NOSPECS and clinical activity score (CAS). Control group composed of euthyroid healthy subjects who did not have any thyroid disorder history.

\section{Methods}

1) Measurement of serum thyroid hormones and

thyroid autoantibodies

- Serum T3, free T4, and TSH

- TR Ab, thyroglobulin antibody $(\operatorname{Tg} A b)$, and

thyroid peroxidase antibody (TPO Ab)

- Serum IgG and $\lg G 4$

2) Ophthalmological evaluation

- NOSPECS' classification system

- CAS

3) Ultrasonographic evaluation

- Hypoechogenicity

- Grade 0 , diffuse high-amplitude echoes throughout whole lobe; Grade 1, low-amplitude and nonuniform echoes in whole or several regions; Grade 2, several sonolucent regions; Grade 3, no apparent echoes or very low amplitude echoes throughout whole lobe.

3. Statistical analysis

All data are expressed as mean \pm standard

deviation (SD). SPSS program version 23.0 (SPSS, Chicago, IL, USA).

\section{Results}

\section{Basic characteristics of GD and control groups}

Sixty four treatment naïve GD patients were enrolled in the study. And 65 euthyroid healthy subjects who did not have any thyroid disease history were enrolled to the control group. Basic characteristics including age, sex, hormone, autoantibodies, $\lg G$, and IgG4 level of GD and control group are summarized in Table 1

2. IgG4 levels were higher in GD with $\mathbf{G O}$ (Table 2, Figure 1)

3. IgG4 levels were significantly higher in the severe form of GO (Figure 2)
Table 1. Basic characteristics between Control and GD groups.

\begin{tabular}{lccc}
\hline & $\begin{array}{c}\text { Control } \\
(\mathrm{n}=65)\end{array}$ & $\begin{array}{c}\text { GD } \\
(\mathrm{n}=64)\end{array}$ & $p$ \\
\hline Sex (M/F) & $14 / 51$ & $18 / 46$ & 0.420 \\
Age (yr) & $47.5 \pm 12.7$ & $37.2 \pm 14.9$ & $<0.001^{\star}$ \\
Family history of AITD [n (\%)] & $15(23.1)$ & $17(26.6)$ & 0.687 \\
Smoking history [n (\%)] & $11(16.9)$ & $12(18.8)$ & 0.822 \\
IgG (mg/dL) & $1178.3 \pm 166.9$ & $1299.7 \pm 320.6$ & 0.135 \\
IgG4 (mg/dL) & $25.4 \pm 12.8$ & $42.0 \pm 25.9$ & $<0.001^{\star}$ \\
IgG4//gG (\%) & $2.2 \pm 1.1$ & $3.3 \pm 1.9$ & $<0.01^{\star}$ \\
T3 (ng/dL) & $105.3 \pm 19.4$ & $288.3 \pm 140.5$ & $<0.001^{\star}$ \\
Free T4 (ng/dL) & $1.20 \pm 0.18$ & $3.37 \pm 1.89$ & $<0.001^{\star}$ \\
TSH (mIU/L) & $2.101 \pm 1.124$ & $0.002 \pm 0.007$ & $<0.001^{\star}$ \\
TR Ab (IU/L) & $0.28 \pm 0.33$ & $13.03 \pm 13.09$ & $<0.001^{\star}$ \\
Tg Ab (IU/mL) & $70.8 \pm 118.7$ & $663.8 \pm 1114.6$ & $<0.001^{\star}$ \\
TPO Ab (IU/mL) & $27.2 \pm 56.9$ & $245.0 \pm 232.4$ & $<0.001^{\star}$ \\
Thyroid size (mm²) & $1132.1 \pm 93.8$ & $1386.5 \pm 270.4$ & $<0.001^{\star}$ \\
Hypoechogenicity & $0.12 \pm 0.33$ & $1.25 \pm 0.47$ & $<0.001^{\star}$ \\
Color Doppler flow & $0.08 \pm 0.27$ & $1.00 \pm 0.69$ & $<0.001^{\star}$ \\
\hline
\end{tabular}

" $p<0.05$. Data are presented as mean \pm SD. GD, Graves' disease; AITD, autoimmune thyroid disease; " $p<0.05$. Dala are pre $\mathrm{Tg} A \mathrm{~b}$, thyroglobulin autoantibody; TPO Ab, thyroid peroxidase antibody.00

Table 2. Comparison between GD without and with GO groups

\begin{tabular}{|c|c|c|c|}
\hline & $\begin{array}{c}\text { GD without } G O \\
(n=42)\end{array}$ & $\begin{array}{c}\text { GD with } G O \\
(n=22)\end{array}$ & $p$ \\
\hline $\operatorname{Sex}(M / F)$ & $16 / 26$ & $2 / 20$ & $0.019^{*}$ \\
\hline Age (yr) & $39.3 \pm 14.2$ & $33.2 \pm 15.7$ & 0.060 \\
\hline Family history of AITD [n (\%)] & $11(26.2)$ & $6(27.3)$ & 1.000 \\
\hline Smoking history [n (\%)] & $4(9.5)$ & $8(36.4)$ & $0.016^{*}$ \\
\hline $\operatorname{lgG}(\mathrm{mg} / \mathrm{dL})$ & $1194.5 \pm 175.1$ & $1500.5 \pm 429.2$ & $0.022^{*}$ \\
\hline $\lg G 4(\mathrm{mg} / \mathrm{dL})$ & $28.9 \pm 13.7$ & $66.9 \pm 25.4$ & $<0.001^{*}$ \\
\hline $\operatorname{lgG} 4 / \operatorname{lgg}(\%)$ & $2.5 \pm 1.3$ & $4.7 \pm 1.9$ & $<0.001^{\star}$ \\
\hline T3 (ng/dL) & $246.5 \pm 99.9$ & $368.1 \pm 171.9$ & $0.002^{*}$ \\
\hline Free T4 (ng/dL) & $2.84 \pm 1.33$ & $4.40 \pm 2.35$ & $0.010^{*}$ \\
\hline TSH (mlU/L) & $0.003 \pm 0.009$ & $0.001 \pm 0.003$ & 0.522 \\
\hline TR Ab (IU/L) & $7.98 \pm 9.06$ & $22.68 \pm 14.31$ & $<0.001^{\star}$ \\
\hline $\operatorname{Tg} A b(I \mathrm{U} / \mathrm{mL})$ & $302.3 \pm 304.3$ & $1353.9 \pm 1669.0$ & $0.005^{*}$ \\
\hline TPO Ab (IU/mL) & $229.9 \pm 237.4$ & $273.8 \pm 225.2$ & 0.589 \\
\hline Thyroid size $\left(\mathrm{mm}^{2}\right)$ & $1376.4 \pm 246.5$ & $1405.8 \pm 316.4$ & 0.702 \\
\hline Hypoechogenicity & $1.17 \pm 0.38$ & $1.41 \pm 0.59$ & 0.069 \\
\hline Color Doppler flow & $0.98 \pm 0.60$ & $1.05 \pm 0.84$ & 0.855 \\
\hline
\end{tabular}

${ }^{*} \mathrm{p}<0.05$. Data are presented as mean $\pm \mathrm{SD}$. GD, Graves' disease; GO, Graves' ophthalmopathy; AITD autoimmune thyroid disease; IgG, immunoglobulin $\mathrm{G}$; $\operatorname{lgG4}$, immunoglobulin $\mathrm{G} 4$; TR Ab, thyroid
stimulating hormone receptor antibody; $\mathrm{Tg} A \mathrm{~b}$, thyroglobulin autoantibody; TPO Ab, thyroid peroxidase antibody.

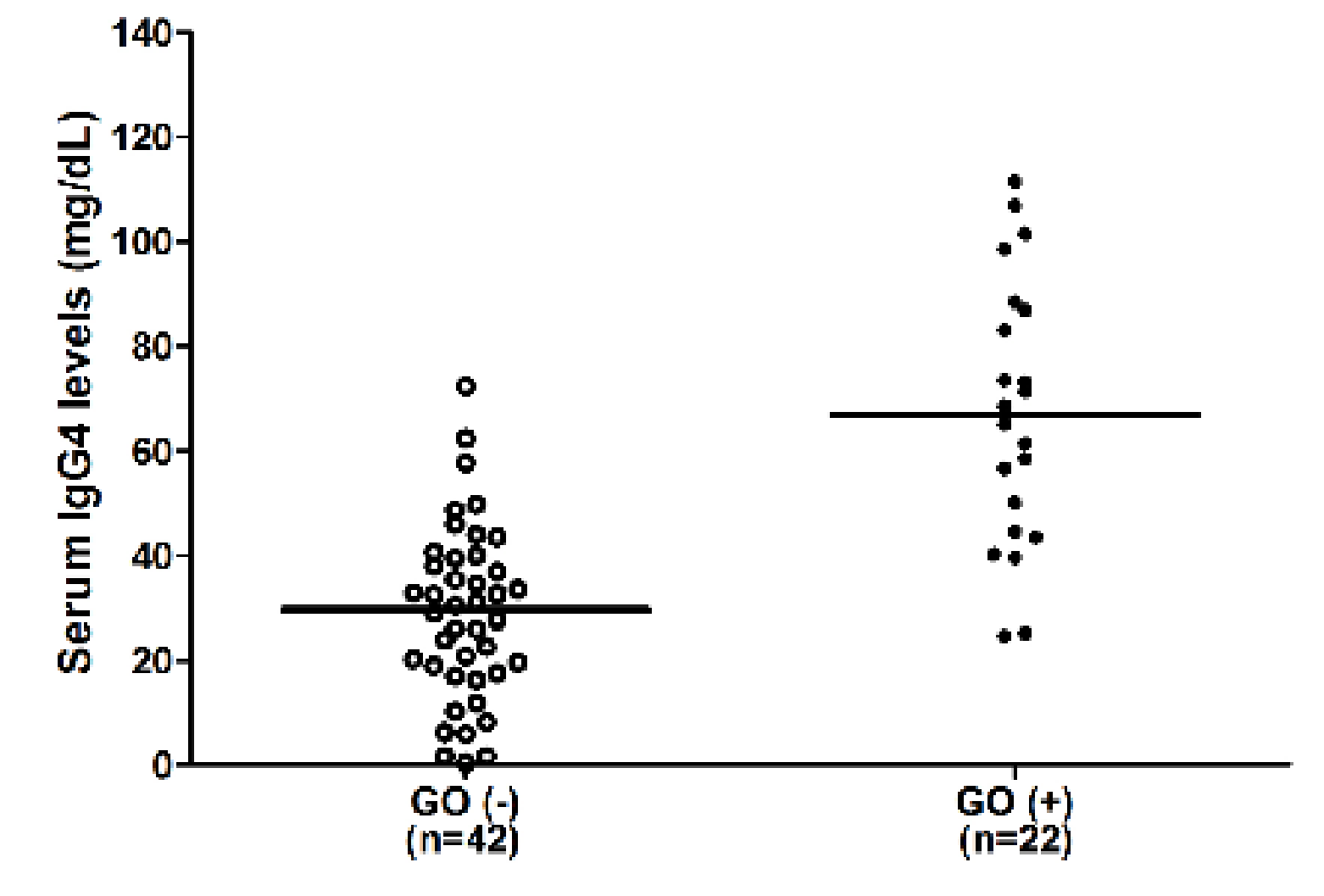

Figure 1. Distribution of IgG4 between GD without and with GO groups.

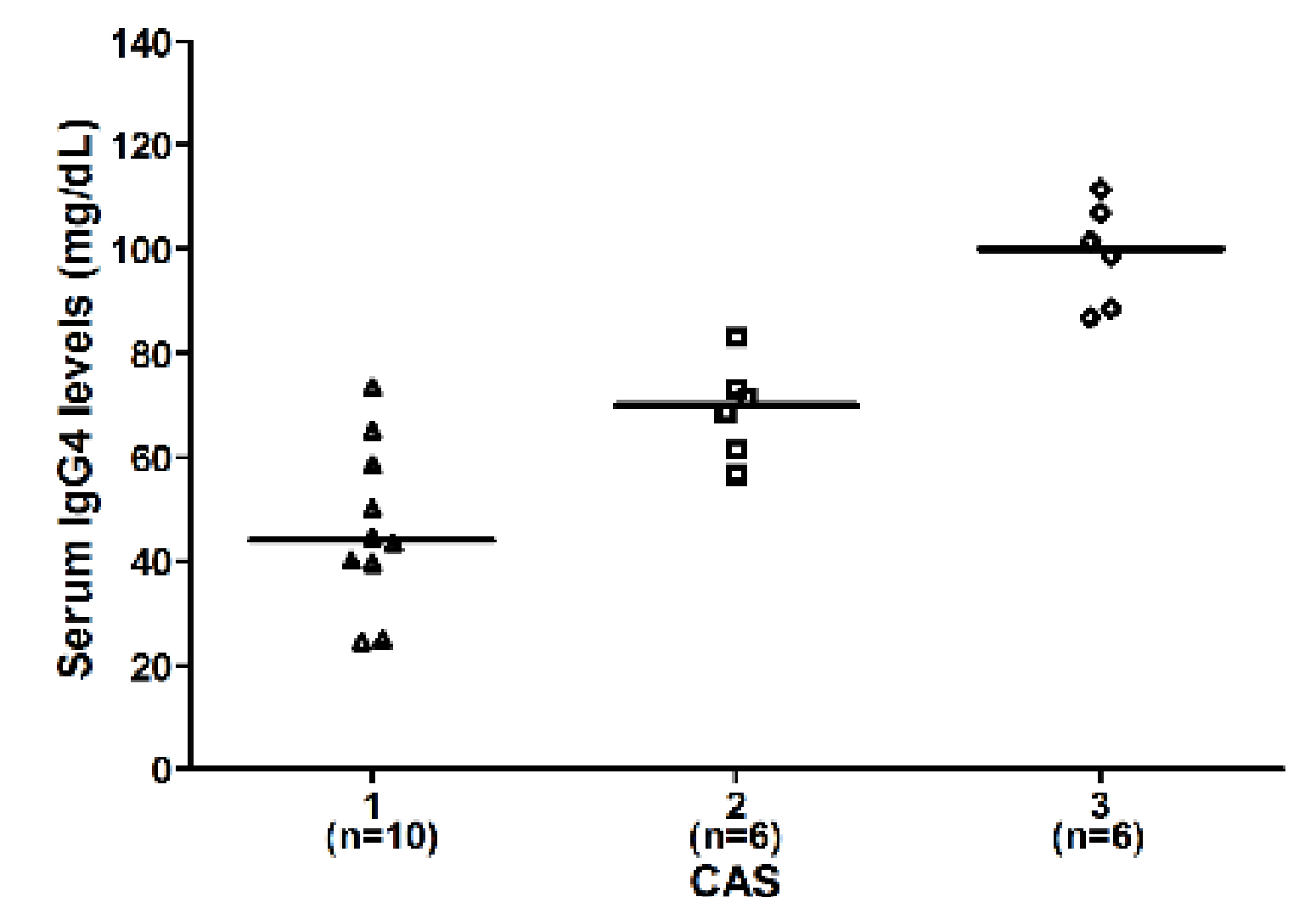

Figure 2. IgG4 levels were significantly higher in the severe form of $\mathrm{GO}$ based on CAS assessments in GD with GO patients
Table 3. Correlation of serum IgG4 levels to clinical and laboratory parameters in GD with GO group.

\begin{tabular}{lcc}
\hline & \multicolumn{2}{c}{$\operatorname{lgG4}$} \\
\cline { 2 - 3 } & $r$ & $p$ \\
\hline Age & 0.067 & 0.767 \\
IgG & 0.063 & 0.779 \\
T3 & 0.185 & 0.411 \\
Free T4 & 0.400 & 0.065 \\
TSH & 0.0 & 1.000 \\
TR Ab & 0.462 & $0.031^{\star}$ \\
Tg Ab & 0.386 & 0.076 \\
TPO Ab & -0.040 & 0.860 \\
\hline
\end{tabular}

'p 0.05. GD, Graves' disease; GO, Graves' phthalmopathy; IgG, immunoglobulin $G$; IgG4, hormone receptor antibody; $\mathrm{Tg} \mathrm{Ab}$, thyroglobulin autoantibody; TPO Ab thyroid peroxidase antibody.

\section{Conclusions}

Serum IgG4 levels are elevated in patients with treatment naïve GD. And IgG4 levels were significantly increased according to the severity of GO. Therefore, measuring serum IgG4 levels in treatment naïve GD may help to provide potential therapeutic options for $\mathrm{GO}$. 